\title{
CRCD Multi-Disciplinary Optical Science and Engineering Program at NJIT
}

\author{
J. F. Federici, A. M. Johnson \\ Dept. of Physics \\ H. Grebel, T. Chang \\ Dept. of Electrical and Computer Engineering \\ R. Barat \\ Dept. of Chemical Engineering, Chemistry, and Environmental Science \\ New Jersey Institute of Technology, Newark, NJ 07102
}

As part of a National Science Foundation (NSF) funded Combined Research/Curriculum Development (CRCD) program, we are developing complementary applied optical science and engineering courses which will (1) provide a unified, multidepartmental optical science/ engineering curriculum and (2) emphasize optics courses which will provide laboratory and classroom training to undergraduate and graduate students in emerging areas of industrial and national importance. In particular, our efforts are focused on the collective strengths of the Engineering School and the Applied Physics Programs: Environmental monitoring and detection of pollutants, industrial process monitoring, optoelectronics, and ultrafast optics and optoelectronics. This multidisciplinary program focuses on optical science and technology as an enabling technology: A technology with applications to many different engineering and scientific disciplines and the potential for significant contributions to those disciplines.

An interdisciplinary team of five professors representing chemical engineering, electrical engineering, and applied physics have developed a unified Optics program at NJIT consisting of three courses with integrated laboratory components. The following courses have been developed:

OPSE 301 -- Optics Principles -- A multidisciplinary introduction to optics principles. OPSE 402 -- Applications -- A multidisciplinary course emphasizing optics applications in Chemical, Environmental, Industrial, and Electrical Engineering and Applied Physics. OPSE 601 -- Advanced Topics -- An multidisciplinary graduate level course.

OPSE 301 and 402, which include integrated laboratory components, are intended for upperlevel undergraduates. OPSE 601, which emphasizes small group projects in each faculty member's research lab, is designed for advanced undergraduate and new graduate students. Detailed course and laboratory descriptions may be found at URL:http//www.njit.edu/Directory/Centers/OPSE.

\section{Motivation}

A 1994 NSF workshop on "Optical Science and Engineering: New Directions and Opportunities in Research and Education" recommended an emphasis in optics research and education because "Optical Science and Engineering is an enabling technology--that is, a technology with 
applications to many scientific disciplines and with the potential to contribute in significant ways to those disciplines." The workshop on Optical Science and Engineering identified a number of critical challenges in Optical Science and Engineering which could lead to significant research and educational opportunities for the programs of NSF. "Research in Optical Sciences and Engineering holds exceptional promise for innovation that will have impact on long-term national goals." Many of those areas highlighted by that workshop review--optical and photonic material and devices, fundamental optical interactions, instrumentation and sensing--are strongly represented in the research of the participating faculty. In addition to its 'enabling' aspects, optics technology represents viable employment potential for new graduates. In an American Institute of Physics survey of initial employment of $1992 \mathrm{Ph} . \mathrm{D}$. recipients, the Education and Employment Statistics Division found that the percentage of graduates who obtained potentially permanent positions differed strongly by subfield: Only $14 \%$ of astrophysicists found permanent jobs immediately after obtaining PhDs, compared with $75 \%$ of those who study optics or lasers. ${ }^{1}$

\section{Laboratory Facilities and Structure}

As part of this program development, 2000 square feet of laboratory space has been renovated to house the new Optical Science and Engineering Laboratory. This student laboratory includes five 4' by 8 ' floating optical tables, separate work benches, He-Ne lasers, optics mounts, lenses, mirrors, photodetectors, spectrometers, and other optical components. As shown in the figure, one section of the lab is equipped with movable chairs for classroom lectures. Students use the work benches for assembling optical components, soldering, sample preparation, etc. Each optical table has its own portable computer equipped with GPIB and RS232 interfaces and a portable equipment rack on wheels for storing electronics such as lock-in amplifiers, oscilloscopes, power supplies, etc.. Wherever appropriate, the laboratory equipment is interfaced

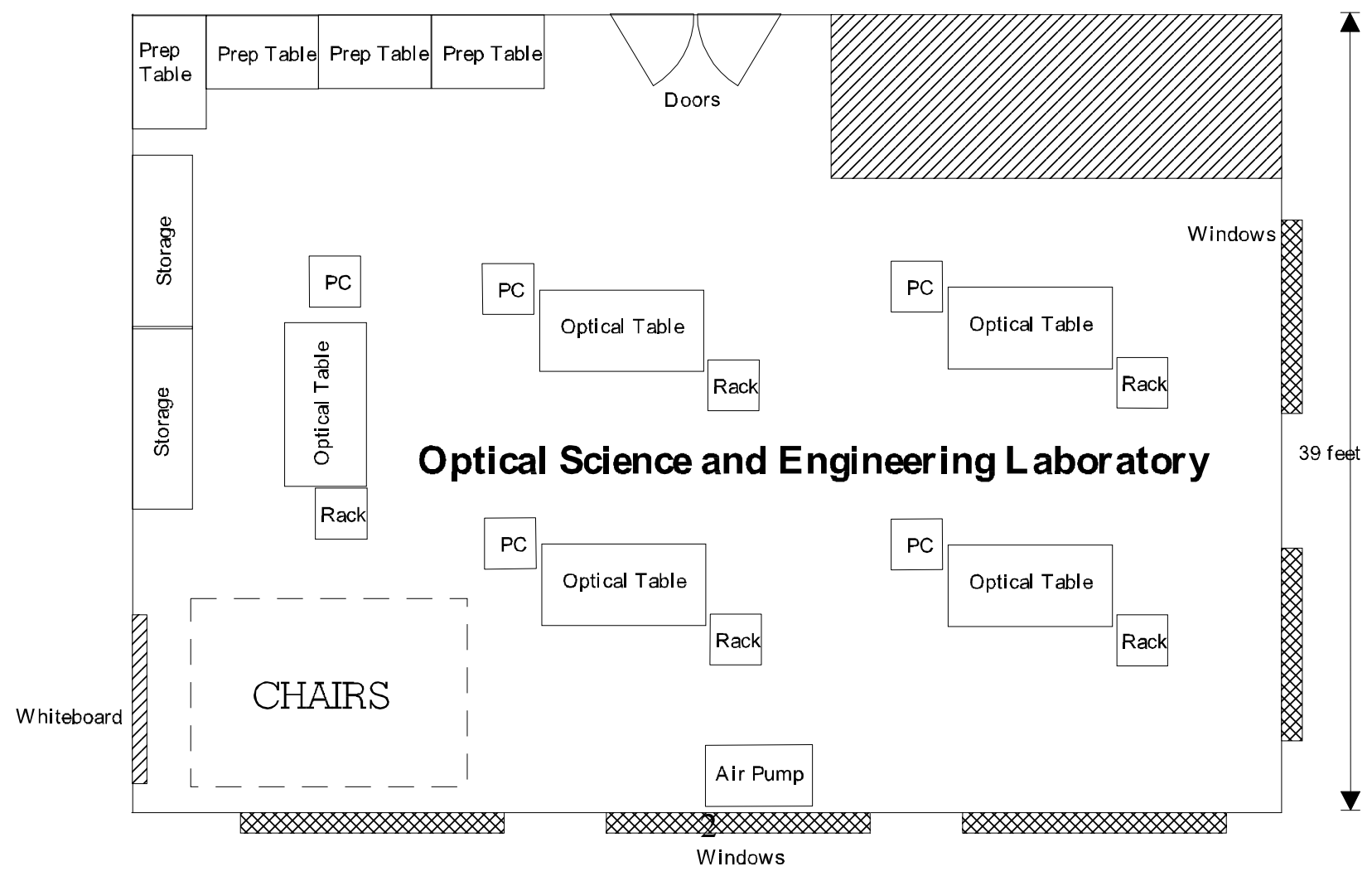


to the computer for easy experimental control, data acquisition, and data analysis. The portability of the computer and equipment rack permits students optimal access to the optical table. In the undergraduate lab, class size is limited to a maximum of two students per optical table (10 students per laboratory section). The purpose of the small class size is to ensure that students get extensive, hands on experience in setting up, diagnosing, and testing optical components and systems. Currently, the only type of laser source used in the undergraduate laboratories is the He-Ne laser which is safe, easy to use, and inexpensive. The graduate student laboratory utilizes advanced laser systems such as excimer, YAG, and Ti:Sapphire lasers.

\section{Curriculum Development}

The following sequence of courses are offered

Optical Science and Engineering 301--Introduction to Optics Principles--offered Fall 96, Spring 97

Optical Science and Engineering 402--Applications--offered Spring 97 Optical Science and Engineering 601--Advanced Topics--offered Fall 96

The following is an overview of the three courses including contributions from all five faculty participants. Updated course outlines and laboratory procedures may be accessed through the OPSE web page URL http://www.njit.edu/Directory/Centers/OPSE.

The curriculum development focuses on the theme of teaching optical science and engineering as an enabling technology. Students will learn not only the fundamental principles of optical science and engineering, but also technologically relevant applications of those principles to optoelectronics, environmental monitoring, industrial process monitoring and position control, and ultrafast optical and optoelectronic phenomena. The students' majors include applied physics, chemistry, chemical engineering, electrical engineering, engineering science, environmental engineering, pre-medical, computer engineering, and computer science. With such a menagerie of student backgrounds, two difficulties arise: (1) scheduling (2) varying levels of mathematical background.

Scheduling -- Due to the multidisciplinary nature of the students and courses, if the OPSE courses were scheduled only during the Spring or Fall semester, a significant number of students would not be able to take the OPSE courses as a free technical course elective. Consequently, OPSE 301 has to be offered during BOTH the Spring AND Fall semesters to accommodate student schedules and demand. Likewise, the class times for each 10 student section is chosen to avoid scheduling conflicts with other departments' core courses.

Mathematical Background -- Due to the multidisciplinary makeup of the students and their differing mathematical backgrounds, the OPSE 301 course does not heavily emphasize the study of optical science based on Maxwell's equations for electromagnetics. OPSE 301, which is predominantly taken by juniors and sophomores, stresses geometric optics and wave optics (e.g. interference and diffraction). Solutions of 
Maxwell's equations are introduced towards the end of the course in conjunction with birefringence and polarization. The de-emphasis of Maxwell's equations allows students of various majors to take the introductory OPSE 301 course without an extensive background in vector calculus and theoretical electromagnetics. However, the OPSE 402 applications course, which is predominantly taken by seniors, incorporates Maxwell's equations and their solutions from the outset.

\section{IIIA. OPSE 301 -- Optics Principles}

The purpose of this course is to provide a survey introduction to optics principles and their elementary applications. It is directed to junior level students in engineering and applied physics.

This course has both lecture and laboratory components. The prerequisites for the course are the sophomore level core-calculus and core-physics courses required of all engineering and science majors at NJIT. A brief outline of the course is given below:

Reference Texts: Fundamentals of Optics, Jenkins and White; Optics, Hecht and Zajac

Prerequisites: Core courses Physics I, II, III, Calculus I -- completed by all sophomores.

LECTURE - Laboratory Safety and Precautions; EM spectrum; Speed of light and propagation; Sources of light: Lasers, Lamps, etc.; Reflection and Refraction -- Snell's law, brewster angle, total internal reflection, dispersion; Geometric Optics -- Mirrors, lenses, magnification, ray tracing techniques and software; Polarization; Birefringence; Interference -- interferometry and thin films; Diffraction -- gratings; Spectroscopy

\section{LABORATORIES}

1. Detection of light (a) Use photodiode and optical power meter to detect laser light. (b) Calibrate neutral density filters/ beamsplitter (c) determine linearity of photodiode.

2. Reflection/refraction (a) study of Snell's law with different materials (b) total internal reflection (measure index of refraction) in a prism.

3. Geometric Optics (a) build a simple microscope (b) expand and collimate a HeNe laser beam (C) spherical/ chromatic aberrations.

4. Ray Tracing (a) Use ray tracing software to study simple combinations of lens, mirrors, and apertures. (b) design eye glasses to correct for astigmatism.

5. Interferometry (a) use HeNe laser and bulk optics to build Michelson interferometer (b) adjust interferometer for circular and localized fringes. (c) measure absolute motion of mirror in one arm of interferometer.

6. Diffraction (a) measure Franhoffer diffraction pattern through slit and compare to theory.

7. Interference Gratings (a) measure diffraction pattern from a grating and compare to theory.

8. Absorption (a) measure absorption of light by various concentrations of laser dye (b) experimentally verify Beers Law.

9. Polarization (a) using a polarized light source, experimentally measure polarization properties of polarizing filters (b) $1 / 4$ wave plate (C) $1 / 2$ wave plate.

10. Polarization by reflection (a) measure ratio of $\mathrm{s}$ and $\mathrm{p}$ polarized light after transmission through a glass plate (b) experimentally determine Brewster's angle. 
11. Light Scattering (a) scattering and absorption of laser light from a suspension of small particles.

12. Spectrometers (a) measure spectral width of He-Ne laser as a function of spectrometer slit size (narrow source compared to instrument resolution) (b) measure spectra of florescent lamp.

For the course, students are assigned homework problems each week in addition to their lab reports. The student laboratory reports constitute of majority of the students' grades. The midterm and final exam have three parts: (1) questions related to homework problems (2) questions related to laboratory assignments and (3) lab-practical questions. The lab-practical questions require the students to examine an optical setup in the laboratory and answer questions concerning the setup. In one question, for example, the students were shown a laser, two polarizers, and a waveplate as illustrated in the figure. By observing the polarization properties of the waveplate, the students were to experimentally determine if it were a $1 / 4$ wave plate or a $1 / 2$ wave plate based on their understating of how waveplates function. Both the midterm and final exams are held in the optics laboratory to facilitate the lab-practical portion of the exams. With five optical tables available, several lab practical setups can be constructed.

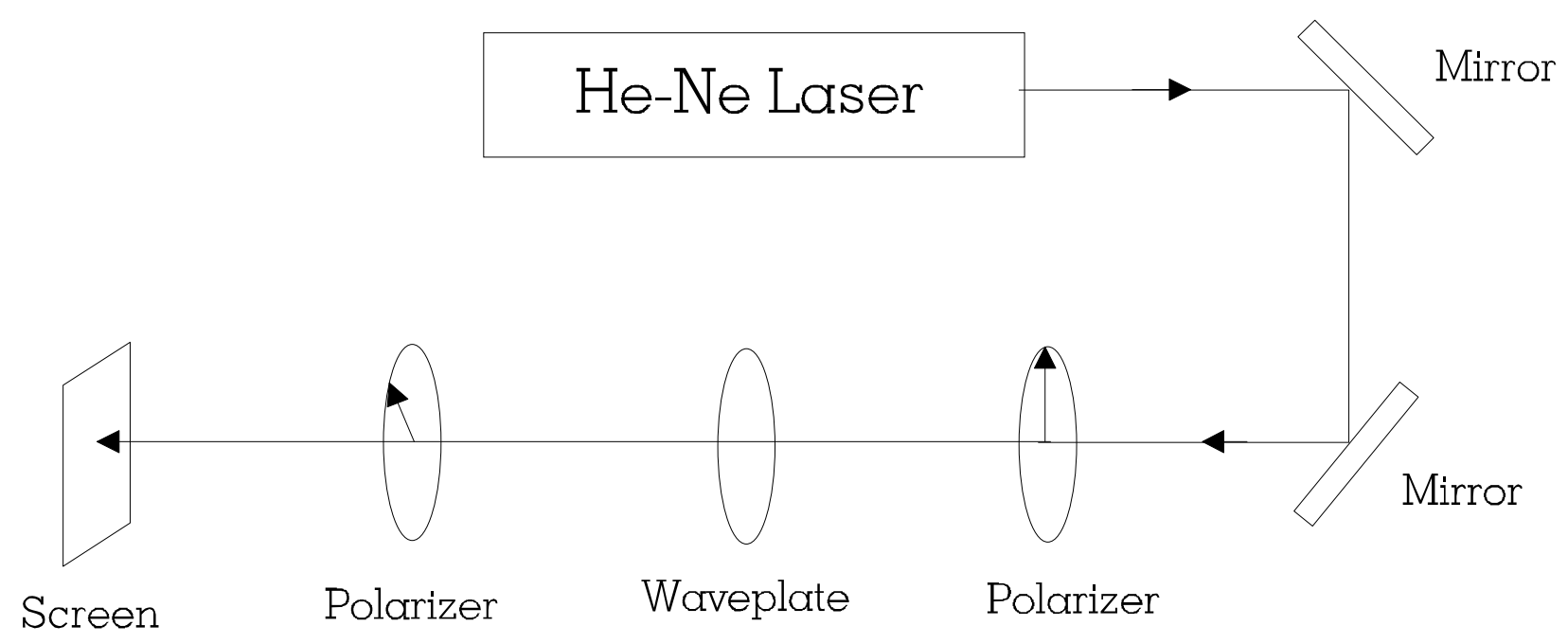

\section{IIIB. OPSE 402 -- Applications}

The purpose of this course is to apply the principles developed in OPSE 301 to selected problems in optoelectronics, sensors, and environmental engineering. OPSE 402, which is to be taken after OPSE 301, is directed to junior/senior level students in engineering and applied physics. The lecture and laboratory portions of the course focus on the different specialties of the associated faculty in which each faculty member designs the experiment/supporting lecture in their field of expertise. Currently, there are four different experiments covering optoelectronics, chemical reactions, environmental monitoring, and motion control/sensing. Eventually, enough laboratories will be developed in order to give the students some choice in available experiments. Given the time constraints of a semester course, the students will eventually be asked to choose 5-6 of the available experiments. This option will allow, for example, Environmental Engineering majors to conduct all of the experiments which are related to their major as well as 
several more to give them a broad, multidisciplinary view of optics as an enabling technology. Likewise, other majors, such as electrical engineers, can focus on laboratories related to their major while conducting experiments in other disciples. This multidisciplinary approach would allow the student flexibility in tailoring their choice of experiments to meet their academic interests and future career path goals.

A brief outline of the course is given below (for Spring 1997 course):

Reference Texts: Environmental Monitoring: Laser Diagnostics for Combustion Temperature and Species, A. Eckbreth; Optoelectronics: Introduction to Lasers and Applications, O'Shea, W. Callen, and Rhodes; Optical Electronics and Quantum Electronics, Yariv; Measurement systems: Application and Design, E.O. Doebelin.; Applied Optimal Control \& Estimation, F Lewis; and recent faculty publications.

Prerequisites: OPSE 301 or permission of Instructor.

LECTURE (current topics) - Laboratory Safety and Precautions; Maxwell's equations: wave propagation; Electro-optics: the change of the refractive index as a function of applied electric; Absorption and transmission; Blackbody radiation; combustion; chemical reaction kinetics; Applications: Fiber Sensors

\section{LABORATORIES (current topics)}

\section{Non-linear Optics}

1. Electro-Optic Devices: Build and test a bulk electro-optic modulator.(4 weeks) Goal: To introduce Electro-Optic Concepts Means: A bar (25x2x2mm) of $\mathrm{LiTaO}_{3}$; An electronic box; Two copper coated breadboards; coaxial connectors; cables; A HeNe Laser; A photolithography kit; High-speed photo diode; Oscilloscope; misc. optics.

Experiment: We will fabricate one electrode on each of the breadboards by use of the photolithography kit. These electrodes will make a transmission line. The line will be formed by pressing each breadboard with electrode against the top and bottom of the $\mathrm{LiTaO}_{3}$ bar. The coaxial connector will feed the RF signal. The modulator will be encased in the box. Two holes will enable input and output of the optical beams. The tests will include phase modulation of the $\mathrm{HeNe}$ laser and assessing the modulation by use of polarizer/analyzer system (AM modulation). The experiment will also include measurements of birefringence using a He-Ne laser, two polarizers, photodectector and oscilloscope.

\section{Environmental/ Chemical Engineering}

1. Batch Chemical Reactor with Laser Diagnostic -- An organic food color dye is a complex organic molecule. These molecules have a broad-banded absorption spectrum. In the case of a He-Ne laser at $632 \mathrm{~nm}$, the dye of choice is one with a spectrum with a reasonably strong absorption at the red $632 \mathrm{~nm}$ line; i.e., blue or green. The transmission of the laser beam through a container of absorbing dye solution is governed by the Beer-Lambert law. In this experiment, applied optics is demonstrated in the monitoring of the aqueous chemical reaction between food 
color (dye) and household bleach (oxidant - sodium hypochlorite). Absorption of a He-Ne laser beam passing through a batch reactor produces data from which global reaction kinetics are determined. Absorbance data will be correlated to determine the "best fit" chemical reaction orders and room temperature rate constant.

2. Flame Temperatures by Interferometry -- Changes in the phase of the light in one arm of an interferometer result in a fringe pattern upon beam recombination. The phase can be significantly affected by passing the beam through a hot gas such as a flame. The refractive index is proportional to the gas density, which is inversely proportional to absolute temperature. Relative flame temperature profiles can be generated by monitoring the changing fringe pattern. This represents a non-intrusive method of flame temperature monitoring, which can be especially important in incineration processes. The fringe pattern profile can be calibrated against radiation-corrected, thermocouple measurements. In this experiment, a small butane/air flame is probed with a Mach-Zehnder interferometer and a type $\mathrm{R}$ thermocouple. The burner is moved systematically relative to the optical axis and thermocouple tip.

\section{Sensor Applications}

1. Motion Sensor - A sensor to measure the velocity of a liquid will be assembled and tested. The sensor will employ optical fibers and a He-Ne laser beam. The objectives include demonstration of the unique utility of optical fibers, especially in their application to small sensors.

\section{IIIC. OPSE 601 -- Advanced Topics}

OPSE 601 is designed to be a research/independent study course which allows the student to interact with the faculty in their research labs in small, focused groups. The prerequisites for the course are OPSE 402 for undergraduates. The course will also be taken by new graduate students. As with the other optics course, this course is multidisciplinary. However, in OPSE 601 the students will study the experimental topics in more detail. Of the available topics, the students must pick three (one month duration) projects to completed during the semester. These projects are to be done in groups of 1 or 2 students in the respective faculty's research laboratories. The small class size will enable individual attention and intensified training in laboratory techniques and research. The students will be required to do preliminary background reading/lectures in their topics of choice, set up the experiment, acquire and analyze data. At the end of 1 month, the student group will rotate to another project. Using this student rotation scheme, the number of students in the laboratories can be kept at a manageable level while the students have the opportunity to use modern optical research tools while conducting their experiments under close guidance of the faculty and associated members (e.g. graduate students, undergraduate assistants, post-docs) of the faculty member's research group. For the course's final exam, the student must present a 15 minute oral presentation on one of the month long projects. For many incoming graduate students, this oral presentation may be the first time that they are required to organize and publicly present their research. The topics are chosen based on their relevance to current research, ability to be completed in the allotted time, and student interest. The list of topics will be constantly updated as research progresses in the respective fields. The topics are a mixture of recently completed and ongoing research. For topics involving ongoing research, several students have made significant contributions towards 
ongoing research. Of the 6 students who enrolled in the first offering of OPSE 601, three of the students will have portions of their work published in journal articles or presented at professional conferences.

Possible topics: (Italics topics offered Fall 1996)

Nonlinear Properties of Materials -- Goal: to teach experimental techniques to measure nonlinear, intensity-related refractive index changes (Kerr type).

Means: Research facilities at the Optical Waveguide Laboratory. The students will use a Z-scan technique to measure the nonlinear absorption and diffraction of silicon nano-clusters. In the experiments, the sample is translated about the focal point of a lens. The far-field is measured using a detector. The far-field along the $\mathrm{z}$-axis of the lens is related to the intensity dependant refractive index changes.

Deposition of nano-size semiconductor clusters -- Goal: to teach thin films deposition and characterization techniques.

Means: Research facilities at the Optical Waveguide Laboratory. The students will use laserablation techniques to deposit films composed of nano-size $(\sim 10 \mathrm{~nm})$ cluster of semiconductor materials ( $\mathrm{Si}, \mathrm{Ge}$, composites). The students will then characterize the films using AFM, x-ray diffraction, raman and linear optical absorption.

Optical Pulse Compression in Single-Mode Fibers -- Equipment: Modelocked Nd:YAG Laser; KTP frequency-doubling crystal; 100 meters of single-mode polarization-maintaining fiber; diffraction gratings; fiber-coupling mounts and microscope objectives; experimental setup for background-free autocorrelation (pulsewidth) [stepper motor, nonlinear crystal, photomultiplier, lock-in amplifier, multi-channel analyzer, etc.]

Experiment: Short optical pulses from a modelocked laser are frequency-doubled and coupled into an optical fiber. Due to the intensity dependent refractive index (Kerr nonlinearity) of the fiber, an intense optical pulse will be spectrally broadened (self-phase modulation) and frequency chirped upon exiting the fiber. The chirped or frequency swept pulse can be reassembled and temporally compressed upon passage through a grating dispersive delay line. Stimulated Raman scattering can also be observed and studied. The degree of compression will be measured by the nonlinear autocorrelation technique.

Concepts: Modelocking; sum frequency generation; nonlinear pulse propagation in fibers; self-phase modulation, stimulated Raman scattering; ultrafast measurement techniques.

Flame Emission as a Pollutant Monitor -- A portable device exists which uses a hydrogen/air flame as an excitation source for atomic or molecular emission. The pollutants could be volatiles containing nitrogen, sulfur, phosphorus, etc. or solutions containing metals. Once optimized, the experiment would call for variation of selected interference filters corresponding to strong flame emission lines of selected pollutants. For a given species, observation of response as a function of pollutant feed concentration would be required.

Detection of Volatile Metal Species by PFF Spectroscopy -- The vapor from a volatile metal compound is probed with an ultraviolet laser beam. Upon absorption of a UV photon, the molecule fragments. Excess energy from the photon results in an excited metal atom, which then 
fluoresces. The fluorescence intensity is proportional to the metal species partial pressure. The experiment would involve variation in measured PFF intensity as a function of metal species vapor pressure (i.e. partial pressure when mixed with an inert like $\mathrm{N}_{2}$ ). Elemental $\mathrm{Hg}$ will be detected by Doppler-shifted resonance fluorescence.

Proximity measurement using intensity modulation. -- Concepts: Intensity based sensors. The Fotonic sensor for distance measurement. The students will build a simple proximity measurement system using a Texas instrument TSL22x digital optical sensor, an IR diode, and a TMS320C26 digital signal processor. A Fotonic sensor will be used to calibrate/verify the experimental results.

Rotation measurement. -- Concepts: Interferometric based sensors. The Sagnac, Michaelson, and Mach Zehnder effects. Phase sensors and the fiber optic gyro. The Sagnac effect will be investigated in a laboratory ring-laser gyroscope set-up which is mounted on a rate table. Effects such as frequency synchronization, scale factor nonlinearity, and bias variations will be addressed. The goal of this project is to exposed the students to industrial sensor (gyro) engineering environments.

Fiber optic sensors -- Concepts: Stress birefringence and microbend. Application to acoustic, pressure, stress, and acceleration sensing. Choice of : measurement of force through stress birefringence, measurement of electric and magnetic fields by electro-optic and magneto-optic effects, measurement of temperature by thermoluminescence and other thermal effects. The students will be introduced to the experimental aspects of fiber optic technology, the associated modulation effects, and optoelectronics interfacing. A TMS320C26 digital signal processor will be used to carry out the necessary real-time processing.

Time-resolved Reflectivity -- The purpose of this experiment is to introduce the students to ultrafast time-resolved reflectivity. The students will use a mode-locked Ti:Sapphire laser, stepper motors, and a time-resolved reflectivity technique to study the lifetime of photogenerated electrons and holes in 'known' materials such as semi-insulating GaAs and low temperature grown-GaAs. Subsequent to the absorption of the Ti:Sapphire light, the photogenerated charge carriers change the real and imaginary indices of refraction at the sample surface. Consequently, the reflectivity of a second pulse of light is altered depending on the time delay between the 'pumping' pulse which generates the carriers and the 'probing' pulse which samples the reflectivity. Using this spectroscopic technique, the students will investigate ultrafast carrier dynamics (lifetime, density) in GaAs (relatively long lifetime) and LT-GaAs (subpicosecond lifetime).

Infrared Quenching of Persistent Photoconductivity in $\mathrm{YBa}_{2} \mathrm{Cu}_{3} \mathrm{O}_{6+x}-$ The purpose of this experiment is to introduce the students to ongoing research in optical properties of superconductivity. The students utilize a wavelength tunable Ti:Sapphire laser and argon laser to alternatively turn on (green light) and quench or turn off (infrared light) the persistent photoconductivity in oxygen deficient $\mathrm{YBa}_{2} \mathrm{Cu}_{3} \mathrm{O}_{6+\mathrm{x}}$ superconductors. The students learn basic cryogenic techniques, photoconductivity measurements, and basic laser operation. 


\section{Summary}

A multidisciplinary optical science and engineering program has been initiated at NJIT. Three courses have been developed: OPSE 301 -- Introduction to Optical Science and Engineering, OPSE 402 -- Applications, OPSE 601 -- Advanced topics. The courses, which emphasize optics as an 'enabling' technology, feature hands-on student laboratories in environmental monitoring and detection of pollutants, industrial process monitoring, optoelectronics, and ultrafast optics and optoelectronics.

For more information, contact J. Federici at federici@admin.njit.edu or visit the optical science and engineering web site at URL: http://www.njit.edu/Directory/Centers/OPSE.

\section{Acknowledgments}

This work is supported by the National Science Foundation, Combined Research/ Curriculum Development (CRCD) program.

\section{BIBLIOGRAPHY}

1.Physics Today, July 1994, p.55

\section{BIOGRAPHIES}

JOHN F. FEDERICI is an Assistant Professor of Physics at NJIT. Professor Federici received his Ph.D. in Plasma Physics from Princeton University. His main research interests are photoinduced superconductivity, optical properties of materials, and chemical sensors.

ANTHONY M. JOHNSON is Professor and Chairperson of the Department of Physics at NJIT and a former Distinguished Member of the Technical Staff at Bell Laboratories (before 1/95). His main research interests are in ultrafast optical and optoelectronic phenomena and he is the Editor-in-Chief of the journal Optics Letters (11/95-11/98). He is a Fellow of the APS, OSA, AAAS, and the National Society of Black Physicists, and a Senior Member of the IEEE.

ROBERT BARAT is an Associate Professor of Chemical Engineering at NJIT. He received his Ph.D. in Chemical Engineering from the Massachusetts Institute of Technology. His current research interests are: Environmental pollutants monitoring, Active process control of high temperature processes, Applied optics in engineering and research, High intensity combustion and incineration, Modeling detailed chemistry in complex reactor configurations, and the Fate of metals in combustion.

HAIM GREBEL is a Professor of Electrical Engineering and Computer Sciences. He received his Ph.D. degree in physics from the Weizmann Institute of Science, Israel. His main research interests are nonlinear optics of nanoclusters, holographic waveguides, and optically controlled microwave devices.

TIMOTHY CHANG is an Associate Professor of Electrical Engineering and Computer Sciences. His main research interests are fiber optic gyroscopes, fiber sensors, and motion control and sensing. 